\title{
Prevalence of problematic smartphone usage and associated mental health outcomes amongst children and young people: a systematic review, meta-analysis and GRADE of the evidence
}

Sei Yon Sohn ${ }^{1}$, Philippa Rees ${ }^{2}$, Bethany Wildridge ${ }^{1}$, Nicola J. Kalk ${ }^{3,4+}$ and Ben Carter ${ }^{5,6^{*}+}$ (D)

\begin{abstract}
Background: Over the past decade, smartphone use has become widespread amongst today's children and young people (CYP) which parallels increases in poor mental health in this group. Simultaneously, media concern abounds about the existence of 'smartphone addiction' or problematic smartphone use. There has been much recent research concerning the prevalence of problematic smartphone use is in children and young people who use smartphones, and how this syndrome relates to mental health outcomes, but this has not been synthesized and critically evaluated.
\end{abstract}

Aims: To conduct a systematic review and meta-analysis to examine the prevalence of PSU and quantify the association with mental health harms.

Methods: A search strategy using Medical Subject Headings was developed and adapted for eight databases between January 1, 1st 2011 to October 15th 2017. No language restriction was applied. Of 924 studies identified, 41 were included in this review, three of which were cohort studies and 38 were cross sectional studies. The mental health outcomes were self-reported: depression; anxiety; stress; poor sleep quality; and decreased educational attainment, which were synthesized according to an a priori protocol.

Results: The studies included 41,871 CYP, and 55\% were female. The median prevalence of PSU amongst CYP was $23.3 \%(14.0-31.2 \%)$. PSU was associated with an increased odds of depression $\left(\mathrm{OR}=3.17 ; 95 \% \mathrm{Cl} 2.30-4.37 ; \mathrm{R}^{2}=78 \%\right)$; increased anxiety $\left(\mathrm{OR}=3.0595 \% \mathrm{Cl} 2.64-3.53 ; \mathrm{I}^{2}=0 \%\right)$; higher perceived stress $\left(\mathrm{OR}=1.86 ; 95 \% \mathrm{Cl} 1.24-2.77 ; \mathrm{I}^{2}=65 \%\right)$; and poorer sleep quality $\left(\mathrm{OR}=2.60 ; 95 \% \mathrm{Cl} ; 1.39-4.85, \mathrm{I}^{2}=78 \%\right)$.

Conclusions: PSU was reported in approximately one in every four CYP and accompanied by an increased odds of poorer mental health. PSU is an evolving public health concern that requires greater study to determine the boundary between helpful and harmful technology use. Policy guidance is needed to outline harm reduction strategies.

Keywords: Problematic smartphone usage, Anxiety, Depression, Sleep, Educational attainment

\footnotetext{
* Correspondence: ben.carter@kcl.ac.uk

${ }^{\dagger}$ Nicola J. Kalk and Ben Carter contributed equally to this work.

${ }^{5}$ Department of Biostatistics, and Health Informatics, Institute of Psychiatry,

Psychology and Neuroscience, King's College London, Denmark Hill, De

Crespigny Park, London SE5 8AF, UK

${ }^{6}$ Cochrane Skin Group, School of Medicine, Nottingham University,

Nottingham, Nottinghamshire, UK

Full list of author information is available at the end of the article
}

(c) The Author(s). 2019, corrected publication 2021. Open Access This article is distributed under the terms of the Creative Commons Attribution 4.0 International License (http://creativecommons.org/licenses/by/4.0/), which permits unrestricted use, distribution, and reproduction in any medium, provided you give appropriate credit to the original author(s) and the source, provide a link to the Creative Commons license, and indicate if changes were made. The Creative Commons Public Domain Dedication waiver (http://creativecommons.org/publicdomain/zero/1.0/) applies to the data made available in this article, unless otherwise stated. 


\section{Background}

Over the past decade there has been an increase in use of smartphones among children and young people (CYP) [1, 2] which has occurred at the same time as a rise in common mental disorders in the same age group, including reported depressive symptoms, poor sleep and suicide ideation [3-5] with grave implications for life-long mental health $[6,7]$ and the healthcare economy [8].

Smartphones became widely available in 2011, since then usage has increased. Smartphone ownership in children aged 11 and older is ubiquitous, and the prevalence of mental health problems peaks during the teenager years [2]. There is a public health uncertainty regarding a possible association between smartphone use and mental health in CYP, and in the UK, policy making has been hindered by a paucity of evidence. Explicitly the debate in the literature has concerned the relationship between amount of screen time, or amount of smartphone use, in CYP and clinically defined, mental health outcomes, with some studies reporting no association and others exhibiting a clear association $[9,10]$. One challenge is the date when the studies were carried out, often before the advent of widespread smartphone use, meaning the term screen-time may include televisions or personal computers, although it has a more common interpretation as a smartphone today [11]. Other limitations include that longer use is assumed as harmful, and this may not necessarily be accurate.

One possibility of the conflicted findings may be that it is not smartphone use per se that is associated with poor mental health, but particular patterns of smartphone-related behaviour. Both the mainstream media and researchers have raised the possibility that people can become addicted to smartphone use, though in the academic realm, this is controversial [12]. Nonetheless, recent years have seen an explosion in research considering the prevalence of problematic smartphone use (PSU), which has been operationalised in such a way that it maps onto concepts of behavioural addiction: tolerance, withdrawal (dysphoria when the battery dies), preoccupation, neglect of other activities, subjective loss of control and continued use despite evidence of harm [13-18]. Other behavioural addictions, such as problem gambling, show robust associations with common mental disorders such as depression [19], where sporadic gambling does not. If a distinctive problematic pattern of smartphone use can be demonstrated to be prevalent, and if this pattern of use is associated with harm, there is value in identifying children and young people with this pattern of use and potentially addressing it clinically. Given the large increase in research studies using tools to estimate the prevalence of PSU (and examine mental health associations), it is now appropriate to evaluate the evidence.

\section{Objectives}

Despite concerns about the impact of smartphones on the mental health and wellbeing of CYP, we are unsure of the prevalence of PSU amongst this cohort, and causal associations between PSU and poor mental health have yet to be established. We therefore undertook a systematic review and GRADE of the evidence with the primary aim of characterising the prevalence of PSU amongst CYP, with smartphones as the exposure, and PSU as the outcome. We also undertook a meta-analysis with the secondary objectives of: assessing sociodemographic characteristics associated with PSU; quantifying the impact of PSU on: mental health outcomes; sleep; and school performance. Mental health outcomes assessed included any reported measure of depression or anxiety (diagnosis or screening questionnaire), and perceived stress; sleep quality. In addition, school performance was included as a measure of functional impairment in this population.

\section{Methods \\ Study selection}

The systematic review was carried out according to the PRISMA statement and reported with the PRISMA checklist [20]; furthermore an a priori protocol is registered on PROSPERO (\#88800). We included randomised controlled trials; cohort; cross-sectional; and case-control studies. Eligibility criteria included studies of mobile device exposure focusing on children and young people (with a mean population age of no greater than 25) [21]. This broader definition of CYP (recently proposed by Sawyer et al.) was specifically chosen, as it is more inclusive of the CYP population who are developmentally vulnerable to problems such as PSU, and also so as to not overlook important data relevant to the paediatric population. Included studies needed to use a scale with a clear threshold to define PSU. Studies that investigated particular uses of smartphones, such as gambling or gaming, were excluded, as these activities have been identified as addictive in and of themselves [22].

\section{Data sources and search strategy}

Searches were carried out from January 1st 2011 to October 15th 2017, with no language restriction. This time restriction was specifically chosen to capture studies of current and modern smartphone technology [23]. A search strategy based on the MeSH headings 'cell phone', 'behaviour, addictive', and 'adolescent' (See Additional file 1: Table S1), was applied to 8 databases, including Scopus, Web of Science, ScienceDirect, PubMed, Medline, CINAHL, PsychInfo, and EMBASE, on October 17th, 2017. Two independent researchers (SS, BW) screened the results from the search strategy, and the 
full texts of all studies that meet these criteria were then further assessed for eligibility. Any disagreements were resolved by discussion with a senior researcher (BC). Additional studies were identified by reviewing the reference sections of relevant papers.

Quality assessment and characteristics of included studies Studies were assessed for methodological quality using a modified Newcastle-Ottawa scale separately for each study design, where each study was assessed and deemed as high, unclear, or low risk of bias across three domains (selection, comparability and outcomes) [24] (See Additional file 1: Table S2). The quality of evidence across the included studies was assessed using GRADE methodology [25]. Study characteristics extracted included: year of study; geographical region; instruments used; response rate; reported prevalence of PSU; mental health; and educational attainment. Study authors were contacted in cases of incomplete data.

\section{Problematic smartphone usage (PSU)}

We defined PSU in accordance with the literature as smart phone use associated with at least some element of dysfunctional use, such as anxiety when the phone was not available, or neglect of other activities $[13,18]$. This was measured by included studies using a range of scales, such as the Smartphone Addiction Scale (SAS) or the Mobile Phone Problematic Use Scale (MPPUS) [13, 14]. We summarise each of the instrument definitions used, and highlight the behavioural domains in Additional file 1:Table S4 and S5.

\section{Data synthesis}

\section{Estimating the prevalence of PSU}

The primary objective was to estimate the prevalence of PSU amongst CYP. The validated thresholds developed by each of the the scales were applied, and this was summarised with a median and interquartile range.

\section{Association between the prevalence of PSU and common mental health outcomes}

The secondary objectives were to investigate PSU associated with the following outcomes: depressed mood; anxiety; stress; poor sleep quality; and educational attainment. A summary of the PSU findings from the studies were assessed using: logistic regression odds ratio (ß); correlation (r); or a Chi-square test.

Where study design, level of exposure of PSU, and outcomes were homogeneous, outcome data were included in a pooled random-effects meta-analysis using the Mantel-Haenzsel method [26, 27]. Where studies reported logistic regression analyses, the analysis data were pooled with dichotomous data using the generic inverse variance method. Pooled odds ratios (OR) are presented with $95 \%$ confidence intervals $(95 \% \mathrm{CI}), p$-values, and $I^{2}$ heterogeneity statistics. Revman 5.3 was used to conduct the analysis.

\section{Assessment of subgroups and statistical heterogeneity} Heterogeneity exceeding 85\% was explored using subgroup analyses [23]. Pre-determined subgroup analyses included: study quality assessment; age; gender; high PSU prevalence ( $>40 \%$ ); time period of study; and geographical region.

\section{Changes since the protocol was registered}

After protocol registration, the following additional outcomes were included: suicidal ideation, and associated psychological factors.

\section{Results}

Identified studies and quality assessment

Of 924 studies identified, 41 studies were included in this review (Fig. 1). Of those, 22 studies were deemed to be of poor methodological quality, and 19 of moderate quality (See Additional file 1: Table S2). Three cohort and 38 cross-sectional studies were included, with 41,871 participants, $55 \%$ of which were female. Included studies were conducted in Europe $(n=9)$, Asia $(n=30)$, and America $(n=2)$ (See Additional file 1: Table S3). There was wide variability in the definitions of PSU (See Additional file 1: Table S4), and the criteria used ranged from a single criterion such as psychological withdrawal phenomena $(n=2)$, to measurement of tolerance, withdrawal, loss of control, preoccupation, neglect of other activities and evidence of harm, which form the criteria for behavioural addictions $(n=19)$ (See Additional file 1: Table S5).

\section{Types of PSU usage}

Communication was the most frequent type of smartphone usage by those with PSU, reported by 14 studies [28-39]. Problematic users reported that social networking was the most important or preferred activity on smartphones [34, 40]. 'Addicted' or 'problem user' groups were linked with particular phone uses: TV watching [35] and social networking $[37,38]$. Lee and Lee [41] found that use of phones to gain peer acceptance was the most significant type of use related to PSU.

Both substance use and other behavioural addictions were associated with PSU. Internet addiction [33, 4244], Facebook addiction [31], compulsive buying [43], increased alcohol use [42], and cigarette smoking [42] were also found to be positively associated with PSU.

\section{Sociodemographic characteristics associated with PSU}

Across 14 studies, age was correlated with PSU [28-33, $40-42,45-49]$, and 17 to 19 year-olds were the most frequent sufferers of PSU. Females were reported as more prone to PSU by 13 studies [31-34, 40, 41, 48-54]; 


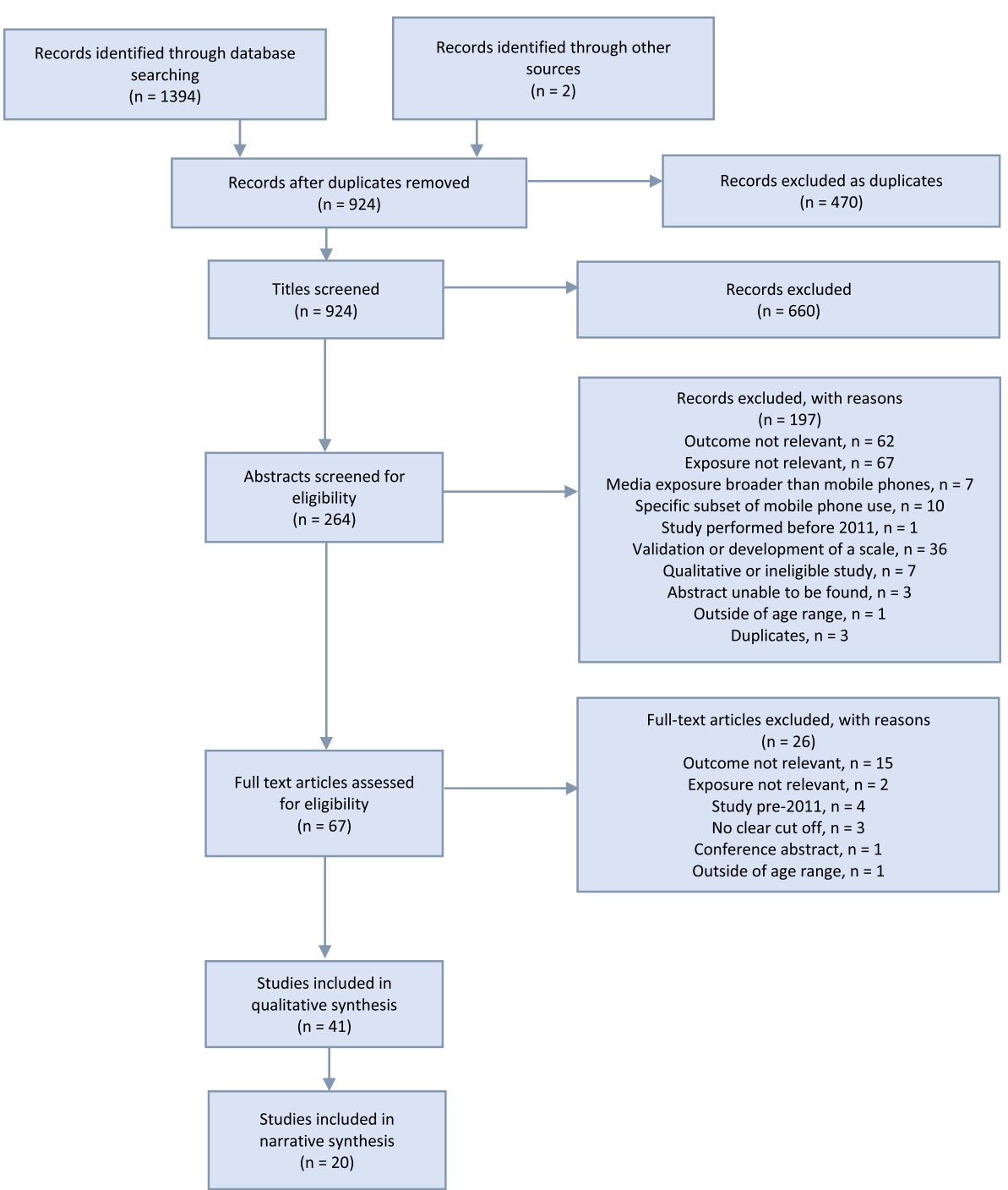

Fig. 1 The PRISMA Flow chart

however 4 studies reported the opposite [35, 46, 55, 56]. PSU in males was correlated with use of media applications and games, while in females it was correlated with communication and social networking applications [28]. PSU was also positively associated with monthly cost of living [28], family income [36], and a higher economic status [42].

\section{Estimating the prevalence of PSU}

Prevalence was assessed using 24 different questionnaires, with the most common being the Smartphone Addiction Scale, Short Version (SAS-SV; $n=7$ ) and the Smartphone Addiction Proneness Scale (SAPS; $n=5$ ), for further details (See Additional file 1: Table S4).

The majority of studies $(n=31)$ found a prevalence between 10 and $30 \%$, and the median was $23.3 \%$ (interquartile range $14-31 \%$, Fig. 2).
PSU associated with mental health outcomes

PSU has been consistently associated with measures of poor mental health, in particular relating to depression, anxiety, stress, poor sleep quality, and day to day functional impairment demonstrated by poor educational attainment. Of the studies included, 20 investigated the relationship between PSU and mental health amongst CYP. This is summarized in a qualitative synthesis (See Additional file 1: Table S6).

\section{Depression}

Eight studies [28, 36, 48, 57-61] reported a significant association between PSU and depression across 10,099 participants. Dichotomous data from four studies was extracted using standard cut-offs for the clinical diagnosis of depression. In those with PSU the odds ratio (OR) of depression was 3.17 (95\% CI, 2.30, to 4.37; $p<0.001$; 


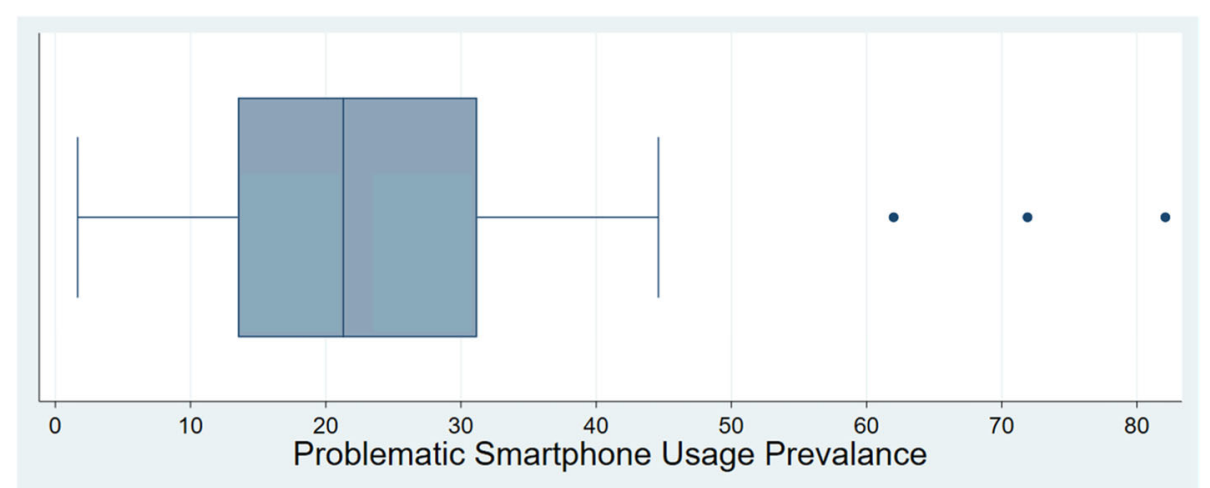

Fig. 2 A boxplot of Problematic Smartphone Usage (PSU) Prevalence

$I^{2}=78 \%$; Fig. 3). Given the consistency of the study findings, we have upgraded this to a GRADE of moderate quality.

\section{Anxiety}

Seven studies [28, 30, 36, 57, 59-61] investigated the relationship between PSU and anxiety in CYP. Of seven studies across 9359 participants, six found a significant positive association between PSU and anxiety; one study reported a negative association [60]. The pooled OR for anxiety amongst CYP with PSU was 2.60 (95\% CI 1.39, to $4.85 ; p<$ $0.001 ; I^{2}=78 \%$ ). The large heterogeneity due to Tavakolizadeh et al. [60], is explained by geography, and the Iranian protests of 2011-2012. After accounting for the heterogeneity, the OR for anxiety amongst CYP with PSU was 3.05 (95\% CI 2.64, to $3.53 ; p<0.001 ; I^{2}=0 \%$;. 3) The GRADE of evidence was categorised as low quality.

\section{Stress}

Five studies [36, 39, 40, 44, 62] investigated perceived stress across 3618 participants. Four studies found a significant association between PSU and perceived stress amongst CYP, whilst Tahtsidou et al. [44] found no significant relationship. A subgroup analysis was introduced due to PSU prevalence. Most heterogeneity was accounted for by Venkatesh et al. [39], a study reporting a PSU prevalence of $71.9 \%$ - this study was subsequently excluded. The pooled OR for typical PSU prevalence and perceived stress amongst CYP was 1.86 (95\%CI 1.24, to $2.77 ; p=0.002 ; I^{2}=65 \%$; Fig. 3 ). The GRADE of the evidence was categorised as low quality.

\section{Sleep}

There were seven studies $[28,35,57,61,63-65]$ which investigated poor sleep across 4194 CYP. Six studies reported a significant positive association between PSU and poor sleep, while Demirci et al. [58] reported no significant association. The pooled OR for the extracted data on PSU and subsequent poor sleep was 2.60 (95\%CI, 1.39, to
4.85, $p=0.003, I^{2}=78 \%$; Fig. 3). The GRADE of evidence was categorised as low quality after accounting for both the narrative analysis and the pooled analysis.

\section{Educational attainment}

Six studies [41, 48, 50, 60, 66, 67] explored PSU and educational attainment across 6655 CYP. Four studies reported a significant association between PSU and poor educational attainment, whilst one [60] found no significant relationship.

Variations in measures of educational attainment were used; it was therefore not appropriate to pool the results of the studies. However, they are summarised to demonstrate the consistency of reported associations between PSU and poor educational attainment (See Additional file 2: Figure S1).

\section{Suicide}

One study reported an increased odds of suicidal ideation amongst those with PSU [62]; however, this was assessed through a single screening question and caution should be taken with this finding.

\section{Psychological factors associated with PSU}

A range of different personality and emotional factors were investigated in relation to PSU. Somewhat paradoxically, traits associated with greater risk-taking (such as low self control, impulsivity, emotional instability, and openness) and traits associated with avoidance of risk taking (such as perfectionism and conscientiousness), were more common amongst problematic smartphone users $[51,52,61,66]$. An insecure attachment style, loneliness $[45,56,65]$, and low self esteem [49] were all associated with PSU.

\section{Discussion}

This is the first systematic review, meta-analysis and GRADE to investigate the prevalence of PSU amongst CYP. The prevalence of PSU amongst CYP was found to be between 10 and $30 \%$, indicating that it is a widespread 

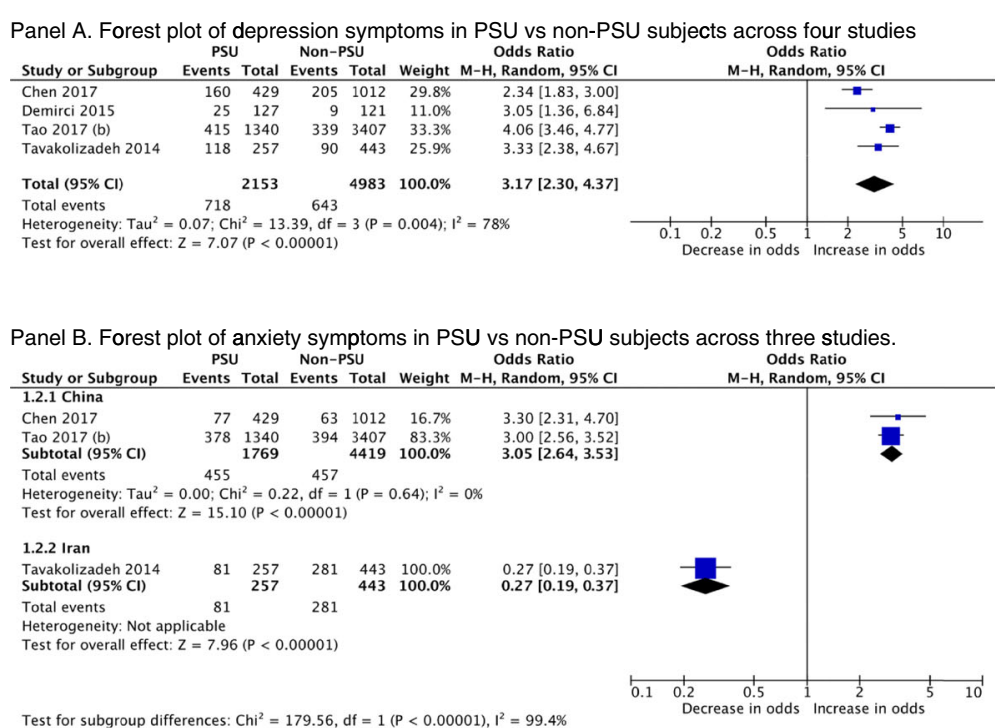

Panel C. Forest plot of perceived stress in PSU vs non-PSU subjects across four studies.

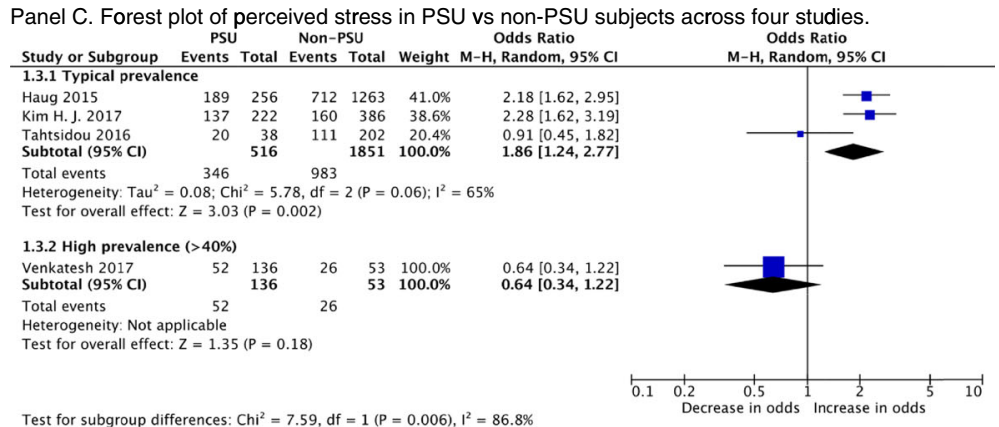

Test for subgroup differences: Chi $^{2}=7.59, \mathrm{df}=1(\mathrm{P}=0.006), \mathrm{l}^{2}=86.8 \%$

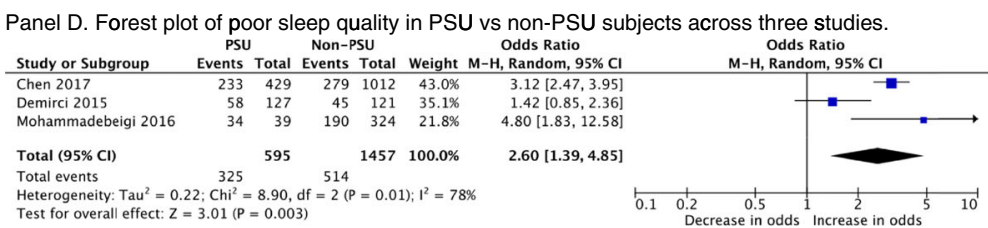

Fig. 3 A meta-analysis of Problematic Smartphone Usage (PSU) and mental health outcomes of: Depression (Panel A); Anxiety (Panel B); Percieved Stress (Panel C); and Poor Sleep (Panel D)

problem. Females in the 17 to 19-year-old age group were most likely to exhibit PSU. Furthermore, PSU was consistently associated with depression, self-reported anxiety, maintenance insomnia, increased perceived stress, and poor educational attainment. Overall, those with PSU had an increased risk of poor mental health, wellbeing and day-to-day functioning.

\section{Context of current literature}

PSU shares many traits with substance abuse disorders and behavioural addictions [13-18], and it appears to be common. This is unsurprising considering that those at risk of PSU have similar traits to those at risk of other addictions. Like alcohol, smartphone use is socially acceptable and widely available. In addition, smartphones are seen to facilitate work and education, as well as leisure. PSU therefore poses a different and arguably much bigger public health problem than substances of abuse or even Internet gaming. The pathogenesis of PSU is poorly understood and likely complex $[45,68,69]$. Some have suggested that the continued interconnectedness and anticipation of response plays a role [23].

The incidence of mental health conditions amongst CYP has increased substantially over the last ten years, representing a significant burden on healthcare systems worldwide $[6,8,70,71]$. The reason for this increase in incidence is unknown, but has been most notable amongst adolescent females, the same cohort shown to be most at risk of PSU in our review [5]. This has parallels between the $68 \%$ increase in self harm rates in the UK since 2011, 
at the same time as the widespread introduction of smartphones [72]. Studies have previously suggested that PSU may at least partly underlie this epidemiological shift. Given the frequency of PSU amongst CYP and its significant association with symptoms of common mental disorders, as highlighted by our review, this relationship and consideration of PSU as a potential causative factor requires urgent further exploration.

\section{Strengths and limitations}

This work is strengthened by the inclusion of studies from wide geographical regions that reported consistent and plausible findings. However, given the nature of the review question, studies were non-randomised and at a high risk of bias. Weaknesses of implementation include varying definitions and thresholds for PSU, some of which were incompletely described. Mental health outcomes were all responses to self-report questionnaires rather than formal diagnoses, suicidal acts or referral to secondary child and adolescent mental health service care, raising the possibility that these are sub-threshold symptoms. Furthermore, reverse causality cannot be excluded as rationale for the associations found.

\section{Implications for policy, practice and research}

Our review indicates that approximately 1 in 4 CYP are demonstrating problematic smartphone use, a pattern of behaviour that mirrors that of a behavioural addiction. A consistent relationship has been demonstrated between PSU and deleterious mental health symptoms including: depression; anxiety; high levels of perceived stress; and poor sleep. Younger populations are more vulnerable to psychopathological developments, and harmful behaviours and mental health conditions established in childhood can shape the subsequent life course. Further work is urgently needed to develop assessment tools for PSU, and prevent possible long-term widespread harmful impact on this and future generations' mental health and wellbeing. In particular, longitudinal studies are required to characterize the causality of the relationships found in this study between PSU and mental health. Possible research could include cohort studies looking at changes in experience of psychopathological symptoms in relation to changes in PSU levels, or a randomized controlled trial comparing the impact of smartphone use, for example in terms of duration or time of day, on mental health outcomes. Future studies should assess the impact of PSU on more objectively evaluated health outcomes, such as depression or anxiety disorders as detected by structured diagnostic instruments (eg the DSM-5 criteria), referrals to secondary mental health services, or primary care psychological therapies services, or prescriptions for medications such as antidepressants.
The prevalence of PSU amongst CYP and its association with symptoms of common mental disorders is a growing public health problem and as such, it should be a concern to policy makers. To address PSU amongst CYP, an accepted and validated diagnostic definition is firstly required, to systematically identify those suffering. Healthcare providers should recognise that excessive or night-time use of smartphones may play a role in the aetiology of mental health and wellbeing problems amongst CYP presenting to their practice. Primary prevention of PSU is difficult given that smartphone use is now a societal norm; however, awareness of the risks of PSU amongst CYP, parents, teachers and healthcare providers could help limit exposure. Further research should develop a consensus regarding the most appropriate diagnostic criteria for PSU, and determine risk factors for PSU. Finally, further exploration of the relationship between PSU and diagnosed mental health conditions is urgently needed to clarify the magnitude of any casual contribution of PSU to the growing burden of mental health conditions amongst CYP.

\section{Conclusions}

Our review indicates that approximately 1 in 4 CYP are demonstrating problematic smartphone use, a pattern of behaviour that mirrors that of a behavioural addiction. A consistent relationship has been demonstrated between PSU and deleterious mental health symptoms including: depression; anxiety; high levels of perceived stress; and poor sleep. Younger populations are more vulnerable to psychopathological developments, and harmful behaviours and mental health conditions established in childhood can shape the subsequent life course. Further work is urgently needed to develop assessment tools for PSU, and prevent possible long-term widespread harmful impact on this and future generations' mental health and wellbeing.

\section{Supplementary information}

Supplementary information accompanies this paper at https://doi.org/10. 1186/s12888-019-2350-x.

\section{Additional file 1: Table S1. Main Search Strategy (from 01/01/2011 to 17/10/2017). Table S2. Characteristics of included studies. Table S3. Quality assessment of included studies using the Newcastle-Ottawa Scale. Table S4. Definitions and Problematic Smartphone Usage terms used, by included studies. Table S5. Mapping the instruments used to assess Problematic Smartphone Usage (PSU) onto criteria for behavioural addic- tion. Table S6. Summary of the results of the included studies.}

Additional file 2 Figure S1. Meta-analyses of Problematic Smartphone Usage (PSU) and the secondary educational outcomes.

Abbreviations

CYP: Children and Young People; PSU: Problematic Smartphone Use

Acknowledgements

Not applicable. 


\section{Authors'contributions}

Conceived the project (BC); Developed the protocol (BC, NK, SS); Carried out the searches (SS, BW, BC), carried out the synthesis (BC, NK, SS), Interpreted the findings ( $B C, N K, P R, S S)$, drafted the manuscript (BC, SS, NK, PR), approved the manuscript (BW). BC is the guarantor of this review. All authors have read and approve the manuscript.

\section{About the authors}

Samantha Sohn, BSc, Medical Student, Institute of Psychiatry, Psychology and Neuroscience, King's College London, UK; Philipa Rees, BSc, MPhil, MBChB, Institute of Child Health, University College London, UK, Academic Clinical Fellow; Bethany Wildridge, BA, Medical Student, Institute of Psychiatry, Psychology and Neuroscience, King's College London, UK, Nicola J Kalk, MBChB MRCPsych PhD, Consultant in Addition Services, Institute of Psychiatry, Psychology and Neuroscience, King's College London, UK; Ben Carter, BSc, MSc, PhD. Senior Lecturer in Biostatistics, Institute of Psychiatry, Psychology and Neuroscience, King's College London, UK.

\section{Funding}

We acknowledge the support of the National Institute for Health Research (NIHR) Biomedical Research Centre at South London and Maudsley NHS Foundation Trust and King's College London (BC, NK). The funder was not involved in the conception, design, analysis or interpretation of this study. During the time the study was done, NK was supported by a NIHR Academic Clinical Lectureship.

\section{Availability of data and materials}

This is an evidence synthesis study, all data is available from the primary research studies, or can be circulated from the corresponding author.

\section{Ethics approval and consent to participate}

Not applicable.

\section{Consent for publication}

Not applicable.

\section{Competing interests}

The authors declare that they have no competing interests.

\section{Author details}

'Institute of Psychiatry Psychology and Neuroscience, King's College London, London, UK. ' Institute of Child Health, University College London, London, UK. ${ }^{3}$ Department of Addictions, Institute of Psychiatry Psychology and Neuroscience, King's College London, London, UK. ${ }^{4}$ South London and Maudsley NHS Foundation Trust, London, UK. ${ }^{5}$ Department of Biostatistics, and Health Informatics, Institute of Psychiatry, Psychology and Neuroscience, King's College London, Denmark Hill, De Crespigny Park, London SE5 8AF, UK. ${ }^{6}$ Cochrane Skin Group, School of Medicine, Nottingham University, Nottingham, Nottinghamshire, UK.

\section{Received: 12 April 2019 Accepted: 31 October 2019}

Published online: 29 November 2019

\section{References}

1. Office for National Statistics. Children's well-being and social relationships, UK: 2018. https://www.ons.gov.uk/peoplepopulationandcommunity/ wellbeing/articles/measuringnmeasuringnmeasur/march2018. 2018. Accessed September 21, 2019.

2. OECD. PISA 2015 Results (Volume III): Students' Well-Being, vol. 2017. Paris: OECD Publishing. https://doi.org/10.1787/9789264273856-en. Accessed September 21, 2019

3. NHS Digital. Mental Health of Children and Young People in England, 2017; Summary of key findings. NHS Digital 2018. https:/files.digital.nhs.uk/A6/ EA7D58/MHCYP\%202017\%20Summary.pdf. Accessed September 21, 2019.

4. Patalay P, Gage SH. Changes in millennial adolescent mental health and health-related behaviours over 10 years: a population cohort comparison study. Int J Epidemiol. 2019. https://doi.org/10.1093/ije/dyz006.

5. Mojtabai R, Olfson M, Han B. National Trends in the Prevalence and Treatment of Depression in Adolescents and Young Adults. Pediatrics. 2016; 138(6). https://doi.org/10.1542/peds.2016-1878.
6. Perou R, Bitsko RH, Blumberg SJ, et al. Mental health surveillance among children--United States, 2005-2011. Morb Mortal Wkly Report Surveill Summ. 2013;62(2):1-35 doi:su6202a1.

7. Whiteford HA, Degenhardt L, Rehm J, et al. Global burden of disease attributable to mental and substance use disorders: findings from the global burden of disease study 2010. Lancet. 2013;382(9904):1575-86. https://doi.org/10.1016/S0140-6736(13)61611-6.

8. Voelker R. Costly mental disorders affect millions of US children and teens. JAMA. 2013;310(1):23. https://doi.org/10.1001/jama.2013.7257.

9. Orben A, Prybylski A. The association between adolescent well-being and digital technology use. Nat Hum Behav. 2019;3(2):173-82. https://doi.org/10. 1038/s41562-018-0506-11.

10. Riehm K, Feder K, Tormohlen K, Crum R, Young A, Green K, Pacek L, Flair $L$, Mojtabai R. Associations between time spent using social media and internalizing and externalizing problems among US youth. JAMA Psychiatry. 2019. https://doi.org/10.1001/jamapsychiatry.2019.2325 [Epub ahead of print]

11. House of Commons Science and Technology Committee. Impact of social media and screen-use on young people's health: Fourteenth report of Session 2017-2019. 2019. https://publications.parliament.uk/ $\mathrm{pa} / \mathrm{cm} 201719 / \mathrm{cmselect} / \mathrm{cmsctech} / 822 / 822 . p d f$. Accessed September 21, 2019.

12. Panova T, Carbonell $X$. Is smartphone addiction really an addiction? J Behav Addict. 2018;7(2):252-9. https://doi.org/10.1556/2006.7.2018.49.

13. Bianchi A, Phillips JG. Psychological predictors of problem mobile phone use. CyberPsychol Behav. 2005;8(1):39-51. https://doi.org/10.1089/cpb.2005.8.39.

14. Kwon M, Kim D-J, Cho H, Yang S. The smartphone addiction scale: development and validation of a short version for adolescents. PLoS One. 2013;8(12):e83558. https://doi.org/10.1371/journal.pone.0083558.

15. Kim D, Lee Y, Lee J, Nam JEK, Chung Y. Development of Korean smartphone addiction proneness scale for youth. PLoS One. 2014;9(5):1-8. https://doi.org/10.1371/journal.pone.0097920.

16. Lin Y-H, Chiang C-L, Lin P-H, et al. Proposed diagnostic criteria for smartphone addiction. PLoS One. 2016;11(11):e0163010.

17. Yildirim C, Correia A-P. Exploring the dimensions of nomophobia: Development and validation of a self-reported questionnaire. Comput Human Behav. 2015;49(Supplement C):130-7. https://doi.org/10.1016/j.chb.2015.02.059.

18. Kwon M, Lee JY, Won WY, et al. Development and Validation of a Smartphone Addiction Scale (SAS). PLoS One. 2013;8(2). https://doi.org/10. 1371/journal.pone.0056936.

19. Bowden-Jones H. Pathological gambling. BMJ. 2017;357:j1593. https://doi. org/10.1136/bmj.j1593.

20. Moher D, Liberati A, Tetzlaff J, Altman DG, Group P. Preferred reporting items for systematic reviews and meta-analyses: the PRISMA statement. PLoS Med. 2009;6(7):e1000097.

21. Sawyer SM, Azzopardi PS, Wickremarathne D, Patton GC. The age of adolescence. Lancet Child Adolesc Heal. 2018;2(3):223-8. https://doi.org/10. 1016/S2352-4642(18)30022-1

22. Potenza MN. Non-substance addictive behaviors in the context of DSM-5. Addict Behav. 2014;39(1). https://doi.org/10.1016/j.addbeh.2013.09.004.Non-substance.

23. Carter B, Rees P, Hale L, Bhattacharjee D, Paradkar MS. Association Between Portable Screen-Based Media Device Access or Use and Sleep Outcomes: A Systematic Review and Meta-analysis. JAMA Pediatr. 2016; 170(12):1202-8. https://doi.org/10.1001/jamapediatrics.2016.2341.

24. Wells GA, Shea B, O'Connell D, et al. The Newcastle-Ottawa Scale Quality Assessment Scale [PDF]. 2013. http://www.ohri.ca/programs/clinical_ epidemiology/nosgen.pd. Accessed 8 Nov 2017.

25. Guyatt GH, Oxman AD, Vist GE, et al. GRADE: an emerging consensus on rating quality of evidence and strength of recommendations. BMJ. 2008; 336(7650):924 LP-926 http://www.bmj.com/content/336/7650/924.abstract.

26. Reeves BC, Deeks JJ, Higgins J, Wells GA. Chapter 13: Including nonrandomized studies. In: Cochrane Handbook for Systematic Reviews of Interventions: Cochrane Book Series. Chichester: Wiley; 2008. p. 389-432.

27. Deeks JJ, Higgins J, Altman DG. Chapter 9: Analysing data and undertaking meta-analyses. In: Cochrane Handbook for Systematic Reviews of Interventions: Cochrane Book Series. Chichester: Wiley; 2008. p. 243-96.

28. Chen B, Liu F, Ding S, Ying X, Wang L, Wen Y. Gender differences in factors associated with smartphone addiction: A cross-sectional study among medical college students. BMC Psychiatry. 2017;17(1). https://doi. org/10.1186/s12888-017-1503-z. 
29. Domple VK, Wadde SK, Gattani PL. Mobile phone dependence among undergraduate medical students in Nanded city. Ann Trop Med Public Heal. 2017;10(1):27-30. https://doi.org/10.4103/ATMPH.ATMPH_71_17 ER.

30. Hussain Z, Griffiths MD, Sheffield D. An investigation into problematic smartphone use: the role of narcissism, anxiety, and personality factors. J Behav Addict. 2017:6(3):378-86. https://doi.org/10.1556/2006.6.2017.052.

31. Lee EB. Too much information: heavy smartphone and Facebook utilization by African American young adults. J Black Stud. 2015;46(1):44-61. https:// doi.org/10.1177/0021934714557034 ER.

32. De MCR. A study of mobile phones and Internet use among Spanish university students. Bordon. 2016;68(3):131-45. https://doi.org/10.13042/Bordon.2016.68307.

33. Garcia-Oliva C, Piqueras JA, Marzo JC. Problematic use of internet, mobile and video games in a sample of adolescents from Alicante. Heal Addict / Salud y Drog. 2017;17(2):189-200. https://doi.org/10.21134/haaj.v17i2.331.

34. Lopez-Fernandez O, Kuss DJ, Romo L, et al. Self-reported dependence on mobile phones in young adults: a European cross-cultural empirical survey. J Behav Addict. 2017;6(2):168-77. https://doi.org/10.1556/2006.6.2017.020.

35. Mohammadbeigi $A$, Absari $R$, Valizadeh $F$, et al. Sleep quality in medical students; the impact of over-use of Mobile cell-phone and social networks. J Res Health Sci. 2016;16(1):46-50 doi:2484 [pii].

36. Long J, Liu T-Q, Liao Y-H, et al. Prevalence and correlates of problematic smartphone use in a large random sample of Chinese undergraduates. BMC Psychiatry. 2016;16(1):408. https://doi.org/10.1186/s12888-016-1083-3.

37. Lee H, Ahn H, Nguyen TG, Choi S-W, Kim DJ. Comparing the self-report and measured smartphone usage of college students: a pilot study. Psychiatry Investig. 2017;14(2):198-204. https://doi.org/10.4306/pi.2017.14.2.198.

38. Tossell C, Kortum P, Shepard C, Rahmati A, Zhong L. Exploring smartphone addiction: insights from Long-term telemetric behavioral measures. Int J Interact Mob Technol. 2015;9(2):37-43. https://doi.org/10.3991/ijim.v9i2.4300.

39. Venkatesh E, Jemal MY, Samani AS. Smart phone usage and addiction among dental students in Saudi Arabia: a cross sectional study. Int $J$ Adolesc Med Health. 2017. https://doi.org/10.1515/ijamh-2016-0133.

40. Haug S, Castro RP, Kwon M, Filler A, Kowatsch T, Schaub MP. Smartphone use and smartphone addiction among young people in Switzerland. J Behav Addict. 2015;4(4):299-307. https://doi.org/10.1556/2006.4.2015.037.

41. Lee C, Lee SJ. Prevalence and predictors of smartphone addiction proneness among Korean adolescents. Child Youth Serv Rev. 2017;77:10-7. https://doi.org/10.1016/j.childyouth.2017.04.002.

42. Tao S, Wu X, Zhang S, Tong S, Hao J, Tao F. Association of alcohol use with problematic mobile phone use and depressive symptoms among college students in Anhui, China. J Public Heal. 2017;25(1):103-12. https://doi.org/10. 1007/s10389-016-0766-Z

43. Jiang Z, Shi M. Prevalence and co-occurrence of compulsive buying, problematic internet and mobile phone use in college students in Yantai, China: relevance of self-traits. BMC Public Health. 2016;16(1):1-8. https://doi. org/10.1186/s12889-016-3884-1.

44. Tahtsidou S, Bellali T, Alikari $\mathrm{V}$, et al. The effect of dependence on mobile phones and computers on the lifestyle and health of nursing students at the University of the Peloponnese. Arch Hell Med. 2016;33(3):355-367 ER.

45. Bian M, Leung L. Linking loneliness, shyness, smartphone addiction symptoms, and patterns of smartphone use to social capital. Soc Sci Comput Rev. 2015; 33(1):61-79. https://doi.org/10.1177/0894439314528779 ER.

46. De-Sola J, Talledo H, Rodríguez de Fonseca F, Rubio G. Prevalence of problematic cell phone use in an adult population in Spain as assessed by the Mobile phone problem use scale (MPPUS). PLoS One. 2017;12(8):1-17. https://doi.org/10.1371/journal.pone.0181184.

47. Jo HS, Na E, Kim DJ. The relationship between smartphone addiction predisposition and impulsivity among Korean smartphone users. Addict Res Theory. 2018;26(1):77-84. https://doi.org/10.1080/16066359.2017.1312356.

48. Kwon YS, Paek KS. The influence of smartphone addiction on depression and communication competence among college students. Indian J Sci Technol. 2016:9(41). https://doi.org/10.17485/ijst/2016/v9i41/103844.

49. Prasad M, Patthi B, Singla A, et al. Nomophobia: a cross-sectional study to assess Mobile phone usage among dental students. J Clin Diagnostic Res. 2017;11(2):ZC34-9. https://doi.org/10.7860/JCDR/2017/20858.9341.

50. Hawi NS, Samaha M. To excel or not to excel: strong evidence on the adverse effect of smartphone addiction on academic performance. Comput Educ. 2016;98:81-9. https://doi.org/10.1016/j.compedu.2016.03.007.

51. Lee H, Kim JW, Choi TY. Risk factors for smartphone addiction in Korean adolescents: smartphone use patterns. J Korean Med Sci. 2017;32(10):16749. https://doi.org/10.3346/jkms.2017.32.10.1674.
52. Leung $\mathrm{CH}$. Assessing mobile phone dependency and teens' everyday life in Hong Kong. Aust J Psychol. 2017;69(1):29-38. https://doi.org/10. 1111/ajpy.12115 ER.

53. Warzecha K, Pawlak A. Pathological use of mobile phones by secondary school students. Arch Psychiatry Psychother. 2017;19(1):27-36. https:// doi.org/10.12740/APP/67931

54. Yildirim C, Sumuer E, Adnan M, Yildirim S, et al. Inf Dev. 2016;32(5):1322-31. https://doi.org/10.1177/0266666915599025.

55. Nikhita CS, Jadhav PR, Ajinkya SA. Prevalence of Mobile phone dependence in secondary school adolescents. J Clin Diagnostic Res. 2015;9(11):VC06-9. https://doi.org/10.7860/JCDR/2015/14396.6803.

56. Yuchang J, Cuicui S, Junxiu A, Junyi L. Attachment styles and smartphone addiction in Chinese college students: the mediating roles of dysfunctional attitudes and self-esteem. Int J Ment Health Addict. 2017;15(5):1122-34. https://doi.org/10.1007/s11469-017-9772-9 ER.

57. Demirci K, Akgönül M, Akpinar A. Relationship of smartphone use severity with sleep quality, depression, and anxiety in university students. J Behav Addict. 2015;4(2):85-92. https://doi.org/10.1556/2006.4. 2015.010.

58. Chen L, Yan Z, Tang W, Yang F, Xie X, He J. Mobile phone addiction levels and negative emotions among Chinese young adults: the mediating role of interpersonal problems. Comput Human Behav. 2016; 55(B):856-66. https://doi.org/10.1016/j.chb.2015.10.030.

59. Tao S, Wu X, Zhang Y, Zhang S, Tong S, Tao F. Effects of sleep quality on the association between problematic Mobile phone use and mental health symptoms in Chinese college students. Int J Environ Res Public Health. 2017;14(2):185. https://doi.org/10.3390/ijerph14020185.

60. Tavakolizadeh J, Atarodi A, Ahmadpour S, Pourgheisar A. The Prevalence of Excessive Mobile Phone Use and its Relation With Mental Health Status and Demographic Factors Among the Students of Gonabad University of Medical Sciences in 2011-2012. Razavi Int J Med. 2014;2(1). https://doi.org/10.5812/rijm.15527.

61. Eyvazlou M, Zarei E, Rahimi A, Abazari M. Association between overuse of mobile phones on quality of sleep and general health among occupational health and safety students. Chronobiol Int. 2016;33(3):293-300. https://doi. org/10.3109/07420528.2015.1135933.

62. Kim HJ, Min JY, Kim HJ, Min KB. Association between psychological and self-assessed health status and smartphone overuse among Korean college students. J Ment Health. 2017;0(0):1-6. https://doi.org/10.1080/ 09638237.2017.1370641.

63. Bhatt N, Muninarayanappa NV, Nageshwar V. A study to assess the mobile phone dependence level and sleep quality among students of selected colleges of Moradabad. Indian J Public Heal Res Dev. 2017:8(1): 41-5. https://doi.org/10.5958/0976-5506.2017.00009.2.

64. Lee JE, Jang S-I, Ju YJ, Kim W, Lee HJ, Park E-C. Relationship between Mobile phone addiction and the incidence of poor and short sleep among Korean adolescents: a longitudinal study of the Korean Children \& Youth Panel Survey. J Korean Med Sci. 2017;32(7):1166-72. https://doi.org/10.3346/ jkms.2017.32.7.1166.

65. Li M, Lu L. The influence of mobile phone addiction on leftbehind middle school students' sleep quality: The mediator role of loneliness. Rev Argentina Clin Psicol. 2017;16(1):71-82. https://doi.org/10.24205/ 03276716.2017 .1006

66. Rajanna SH, Sharma MK, Palanichamy TS. Exploration of Technology use Pattern among Teenagers and its Relationship with Psychological Variables. ASEAN J Psychiatry. 2016;17(2):239-249 ER.

67. Wang C, Wang SY, Li WH, Dong XM, Chi GB. Study on the mobile phone dependence syndrome and its distribution among 2213 college students in Guangzhou. Zhonghua Liu Xing Bing Xue Za Zhi. 2013;34(10):949-52.

68. Billieux J. Problematic use of the Mobile phone: a literature review and a pathways model. Curr Psychiatr Rev. 2012;8(4):299-307. https://doi.org/ 10.2174/157340012803520522.

69. Wang J-L, Wang H-Z, Gaskin J, Wang L-H. The role of stress and motivation in problematic smartphone use among college students. Comput Human Behav. 2015;53(Supplement C):181-8. https://doi.org/10.1016/j.chb.2015.07.005.

70. Bardach NS, Coker TR, Zima BT, et al. Common and costly hospitalizations for pediatric mental health disorders. Pediatrics. 2014;133(4):602-9. https:// doi.org/10.1542/peds.2013-3165.

71. Shaffer D, Fisher P, Dulcan MK, et al. The NIMH diagnostic interview schedule for children version 2.3 (DISC-2.3): description, acceptability, prevalence rates, and performance in the MECA study. Methods for the 
epidemiology of child and adolescent mental disorders study. J Am Acad Child Adolesc Psychiatry. 1996;35(7):865-77.

72. Morgan C, Webb R, Carr M, Kontopantelis E, Green J, Chew-Graham C, Kapur N, Ashcroft D. Incidence, clinical management, and mortality risk following self harm among children and adolescents: cohort study in primary care. BMJ. 2017;359:j4351.

\section{Publisher's Note}

Springer Nature remains neutral with regard to jurisdictional claims in published maps and institutional affiliations.

Ready to submit your research? Choose BMC and benefit from:

- fast, convenient online submission

- thorough peer review by experienced researchers in your field

- rapid publication on acceptance

- support for research data, including large and complex data types

- gold Open Access which fosters wider collaboration and increased citations

- maximum visibility for your research: over $100 \mathrm{M}$ website views per year

At BMC, research is always in progress.

Learn more biomedcentral.com/submissions 